\title{
MENTAL DISORDER IN CUSHING'S SYNDROME
}

BY

\author{
W. S. MACLAY AND A. B. STOKES \\ Pathological report by D. S. RUSSELL
}

From the Maudsley Hospital and Bernhard Baron Institute of Pathology, The London Hospital

(Received 16Th February, 1938)

RESEARCHES into endocrine function and dysfunction have thrown new light on many clinical conditions, and great hopes have been aroused that obscurities of causation may ultimately be traced to an endocrine source. The obvious relationship between certain emotional states, e.g. fear, and the activity of certain endocrine glands, e.g. suprarenals, suggested that such an approach might be specially valuable in psychiatry; nevertheless, the specific association of a given mental disorder with a given endocrine state has not been accepted and has fallen into disrepute. Perhaps because of this certain aspects of endocrine disorder have not been sufficiently stressed.

An endocrine disorder may be related to an abnormal mental state in the following ways : (1) Both the endocrine and mental illness may be expressions of an underlying cause, either of a pathological genetic disposition or an environmental noxa. (2) The endocrine disorder may precede and be the immediate cause of disturbed function in the brain. (3) The abnormal mental state may precede and be the cause of abnormal endocrine function. (4) The endocrine disorder may produce certain systemic disturbances which secondarily involve the brain structure and disturb its function. (5) The endocrine disorder may produce bodily changes of social significance so great that the personality shows signs of stress. These relationships, easily differentiated in theory, are in practice obscure, and the obscurity is made greater by the possibility of vicious circles in which the endocrine and neural factors influence one another successively.

In considering the mental states associated with Cushing's syndrome, the fifth and fourth categories of relationship, as made above, are of obvious importance. The social consequences of this syndrome in any patient are tremendous, but particularly so in females. Obstinate hirsuties, adiposity, and disturbances of sexual function are outstanding as social obstacles to which the patient has to make a new adjustment. Again, owing to metabolic changes, hypertension, arteriosclerosis, possible cardiac and renal failure, etc., the adjusting mechanism can itself become impaired and this impairment may 
become manifest as a change of personality. In these circumstances it would be surprising if symptoms of mental illness, of greater or lesser degree, were not present in a large proportion of patients. However, out of 49 cases in the literature of Cushing's syndrome, including tumours of the suprarenal cortex and thymus, the mental state was mentioned in only 20 . It is unnecessary to give full references to the literature of Cushing's syndrome, as it has recently been well reviewed by Pardee (1937), who gives 152 references. The main abnormal findings were stated as follows :-

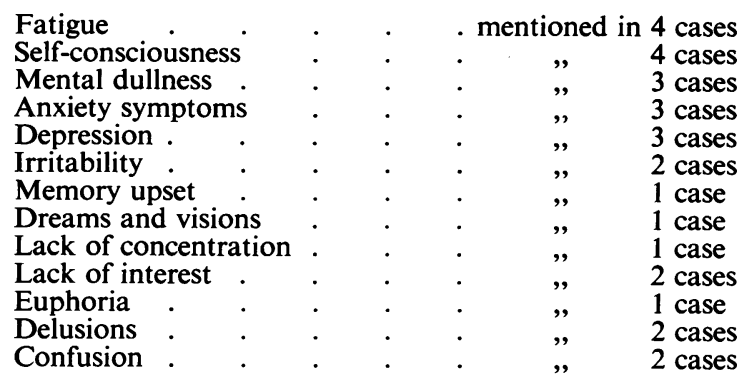

In such reports, the mental status was, with few exceptions, only referred to in casual sentences or short paragraphs from which the above data were compiled, but from their content there was clear evidence of the presence of a mass of interesting psychiatric material which had been disregarded.

The following case illustrates the development of a psychosis in a victim of this disorder, in whom the opportunities for observation and investigation were exceptional in that she was under medical care during nearly the whole of her illness, and was in hospital with daily observation for the last two years of her life.

\section{Personal Case}

Female, æt. 29 years. Was admitted to hospital in May 1935 because of two suicidal attempts following a period of depression and change of personality. She was under observation in hospital until her death in April 1937.

Her father, æt. 75 years, and mother, æt. 64 years, are alive and well. The father has been married twice, having one daughter by his first marriage and two sons and four daughters, of whom the patient is the second, by his second marriage. The family life has been happy and normal. There is no history of any nervous, mental, or endocrine illness among the relatives.

Her early development was uneventful. She did well at school, where she matriculated and won a scholarship. She enjoyed her school life, liked games and was good at them. Since then she has worked efficiently as a clerk for four years in one post and nihe years in another, earning fifty shillings a week. She worked until one week before admission to hospital. Menstruation started at 16 years and was regular and normal until the onset of her illness. Her health has been good except for an attack of influenza in 1918. There is no history of any previous mental illness. Her previous personality has been good. Her friends often called her placid and carefree. She got on well with her family and friends, although in her own opinion she was inclined to be shy and sensitive. She played a lot of games, attended evening classes, and was fond of reading. She was never jealous and never worried about her health. Her appearance was attractive, as shown in the photograph (Fig. 1). 
She was perfectly well until August 1933, when she developed amenorrhœa. From then until her admission she only menstruated on three or four occasions, the flow being very scanty. About the same time she showed excessive growth of hair on the face, some brown pigmentation, and severe pain in the lower abdomen. She was admitted to a hospital in March 1934 for the above complaints, where investigations revealed only an irregular glycosuria. In November 1934 she complained of spots in front of her eyes and pain with tenderness on the left side of her face. During the latter part of 1934 and in 1935 there was a gradual but marked change in her personality. She became seclusive, refusing all social engagements ; she became morbidly sensitive about the hair on her face ; she became jealous of her sister and of any new clothes that her sister bought. She gradually lost interest in everything, could not concentrate, and became depressed. For some months before admission she felt hopeless and had fits of weeping. She would wake up at night and cry, "Why can't I die ?" and on one occasion tried to throw herself out of the window. She was able to continue work until May 1935, but was found one day to have inflicted superficial wounds on her wrists with a razor. She was admitted to an observation ward from which she was transferred to the Maudsley Hospital.

She was restless, self-concerned, and unhappy, but discussed her illness intelligently. Her mood was one of bitterness against the world, and she attributed her depression to worry about her appearance and the apparent inability of any doctor to cure her. She thought that all her symptoms might be due to anæmia. She was insistent that she had no desire to live unless cured. There were no delusions or hallucinations ; she was correctly orientated; her memory showed no apparent defect, though she herself thought that it was bad. Her grasp of general information was good, but she showed poor powers of concentration and had a real difficulty in doing an arithmetical test, such as subtracting serial sevens. Her insight was poor and she seemed unwilling or unable to understand a simply worded explanation of her illness. She would accept reassurance temporarily, but asked all the same questions again on the following day. Her chief complaints were of nausea and anorexia, hair on the face, and pain in the region of the left temple. On questioning her she had many other symptoms, namely, amenorrhœa, blurring of vision, with black spots in front of her eyes, backache, shooting pains in the lower abdomen, and a feeling of fatigue and weakness which she described by saying, "I used to walk quickly ; now I amble along." Most of the above symptoms persisted during her stay in hospital.

She was a well-built woman with a tendency to adiposity of the face, neck, and trunk, but not of the limbs. The face looked congested and plethoric, while the limbs showed a "cyanotic" appearance. There was marked hypertrichosis affecting the face, body, and limbs, especially the face, where the hair on the upper lip and chin was curly and grew rapidly, requiring frequent shaving or pumice-stone treatment to control it (Fig. 2). She bruised easily and even without injury small ecchymoses often appeared on various parts of her body. At one stage of her illness numerous petechial spots appeared on both breasts. The visual fields were normal. Ophthalmoscopic examination showed no abnormality. The blood pressure, which was recorded at frequent intervals, was always raised and was about 180 systolic and 110-120 diastolic. The left ventricle was enlarged and the aortic 2 nd sound accentuated.

The laboratory findings were as follows : (1) Blood : (a) Complete blood examinations were made at intervals, but never showed any tendency towards polycythemia or any marked variations from the example given. Red blood corpuscles, 4,440,000 ; white blood corpuscles, 6,000; hæmoglobin, 14.3 gm.; colour index, 1.0. Size, $7 \cdot 2 \mu$. Differential count : polymorphs, 4,260; lymphocytes, 1,500; hyaline, 180 ; basophils, 60 . (b) Blood urea, $21 \cdot 2 \mathrm{mg}$. per cent. (c) Serum calcium, 10.0 mg. per cent. ; serum potassium, $15.0 \mathrm{mg}$. per cent. ; serum sodium, $226.0 \mathrm{mg}$. per cent. ; phosphatase, 0.274 units. (d) Coagulation time $=3$ minutes (Sabraze's Method). (e) W.R. negative. ( $f$ ) Fasting blood-sugar level was always very high (Calvert's modification of Folin and Wu), as shown in graph of blood sugar curve. (2) The 
cerebrospinal fluid was normal when examined on two occasions. (3) Basal metabolic rate, +19 per cent. (Benedict's method) ; impedance angle, normal. (4) Zondek Ascheim test, negative. (5) Urine consistently showed a high specific gravity and much sugar except when insulin was being given. In spite of the large quantities of sugar, up to as much as $5.0 \mathrm{gm}$. per cent., there were never any ketone bodies in the urine. Traces of albumen only appeared when the urine was infected. The glycosuria was easily controlled by 10 units of insulin twice a day. A urea clearance test done in August 1935 was the only indication that the kidneys might not be acting efficiently. Urea clearance test : blood urea, $21.2 \mathrm{mg}$. per cent. After first hour, 64.5 per cent. ; after second hour, 26.0 per cent. (6) X-ray appearances : The skull and pituitary fossa were X-rayed on four occasions without showing any abnormality; the last

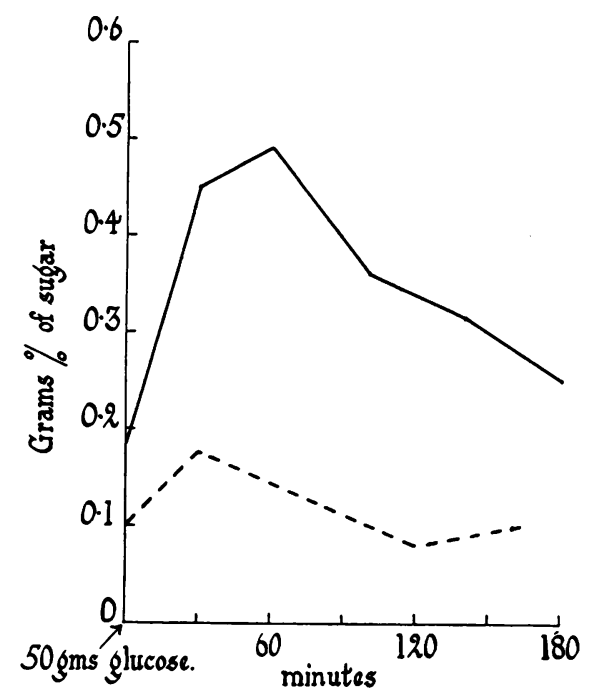

photograph showed the decompression opening (referred to later) and the radon seeds, one of which was in the pituitary fossa. Radiographs of the long bones and spine were normal on admission, but a year later definite osteoporosis of the spine was seen. Radiographs of the chest showed an enlarged left ventricle of the heart. There was no evidence of an enlarged thymus.

In September 1935 a course of deep X-ray therapy directed at the pituitary gland was commenced, but had to be interrupted after two treatments owing to the patient falling in the ward and striking the right side of her head on the floor. This was followed by papillodema, thrombosis of the central vein of the retina, and numerous retinal hæmorrhages in the right eye. The condition improved slowly, but left her with marked defect of vision in the right eye. The left eye was not affected. In December 1935 a laparotomy was done and an appendix which showed signs of chronic inflammation both by naked eye and microscopic examination was removed. The surgeon reported that the uterus was infantile, the ovaries both very small, and that nothing abnormal could be felt in the region of the suprarenal glands. Two days after the operation she suddenly became unconscious and remained so for two days, the condition being suggestive of a small cerebral embolus. From this she made a good recovery. Up to this time she had shown increasing self-concern and depression, remarking on several occasions that she would commit suicide if it were not for the trouble to others which such an action would cause. At the beginning of 1936 her mood seemed to vary with the pain in the left temple. When free from this pain she was up and about, behaving in a cheerful, sociable way with the other patients, but about March she became worse again, was very preoccupied with her ailments, and 
for the first time began to express morbid ideas about her bodily state, saying, "I'm certain there is something wrong with my bowels" or "I'm sure there is something behind my eye."

The course of deep X-ray therapy was recommenced and completed without any untoward effects, except loss of hair from the parts of the head irradiated. By May she was the picture of misery ; delusions had developed and were characterized by feelings of self-reproach. She would ask if she was a "bad woman" of if she was really a thief or if she had committed some crime. She had vague ideas that her amenorrhœa might be connected with pregnancy. She was suspicious and rather bewildered ; she thought there might be a dictaphone in the room to spy on her and had the feeling at night that there was play-acting going on in the ward, and that the whole of her life was re-enacted. She took her food poorly and lost nearly a stone in weight.

In June 1936 it was decided to explore the pituitary fossa. Mr. Hugh Cairn's operation note is as follows : " The pituitary was approached via the right transfrontal route without any special difficulty, in fact, more easily than usual, because when the dura was opened the subarachnoid space was found to be greatly distended with fluid all over the right frontal region. The cortex itself, however, did not appear to be shrunken or atrophic. The exposure of the pituitary was technically very difficult, though division of the right optic nerve helped a little. A small gland in a small sella was seen and it was possible to insert two radon seeds. She recovered from the operation satisfactorily."

After the operation there was little change in her mental state. She was still restless and uncertain and needed constant observation. She admitted that her headaches were a little better, but was concerned about her eyesight. She was now quite blind in the right eye and had a left temporal hemianopia. She was sent as a voluntary patient to a mental hospital in October, 1936. During the remainder of her illness she was at most times self-reproachful, deluded, and impulsive. Ideas of reference were marked, she thought that she heard people say " that woman " in a scornful way, and felt that her name was coupled with unpleasant things. She thought that she was accused of theft and that people avoided her. Sometimes she was noisy, laughing, talking, singing, and trying to run away. Occasionally she was confused, but often it was possible to get her to talk coherently and to discuss her illness, though she constantly reverted to her delusions. The degree of blindness in the left eye increased with the development of marked optic atrophy. It was difficult usually to get her to take her diet, excepting at the period shortly before her death, when she ate voraciously. She had increasing thirst, which was never satisfied. She died in April 1937.

\section{Discussion}

The post-mortem findings are described and discussed in the appendix, but even without them the clinical picture is highly suggestive of Cushing's syndrome. The symptomatology conforms closely to the requirements laid down by Cushing, including as it does weakness, fatigability, backache, abdominal pains, aching pains in the eyes, retinal hæmorrhages, ecchymoses, pigmentation, plethoric appearance of skin, abnormal hirsuties, adiposity of face and shoulders, sexual dystrophy, vascular hypertension, osteoporosis, hyperglycæmia, polyphagia, polydipsia, and susceptibility to pulmonary infection. Cushing (1932) himself recognized that the syndrome is polyglandular; and a polyglandular syndrome is apparently nothing more than a group of secondary functional alterations occurring in the ductless gland series whenever the activity of one of the glands has become primarily affected. It may, however, be impossible 
to determine clinically where the primary fault is located. Lescher and Robb Smith (1935), after a careful comparison of the pituitary basophilic syndrome and the adrenal cortico-genital syndrome, say: "It must be concluded that Cushing's syndrome, complete in its entirety, may be caused by such diseases as tumours of the adrenal cortex, and so a clinical distinction cannot always be made between them."

The difficulties of establishing the nature of the relationship between the endocrine state and the mental disorder are illustrative of the difficulties always arising in this field. The hyperthyroid state is associated clinically with anxiety and irritability; the cretin has been described as showing psychic and motor retardation, not infrequently accompanied by obsessional features ; pancreatic tumours have been associated with neurasthenic symptoms of widely varying nature ; hypoparathyroidism has been complicated by hallucinatory experiences; gonadal pathological changes have been described in schizophrenia, etc. In regard to pituitary disorders Birnbaum (1930) says : "The mental states accompanying anomalies of function or disease of the hypophysis should be regarded with special reserve. First, some of these pituitary disorders are still debatable in their pathological interpretation, and secondly some of the observations are based on tumours which produced signs of increased intracranial pressure." Kraepelin and Lange (1927) after describing some of the mental changes which may accompany acromegaly go on to say: "Furthermore, in acromegaly psychoses can be observed that are in no close relation to the physical disease . . . it is most likely in such instances that there is only an incidental combination which is favoured by a common predisposition"; or again, after mentioning the wide variety of mental disturbances which can occur with Frohlich's syndrome, they say : "It is an open question how far these observations point to a causal relationship to the hypophysical disease ; it is most likely for the confusional states and for the dementia and somnolence which can be observed in the later stages of the disease." Such relationships as have been described are complex and as difficult to elucidate as in the present case. Hoskins (1934) sums up the position when he says: "Psychoses may be importantly determined by antecedent or concurrent endocrine abnormality, but none of the evidence compels that conclusion."

The first evidences of ill health in this patient were physical complaints, amenorrhœa, changed appearance, hypertrichosis, pain in head and abdomen, of such a character as to make the change of personality which followed easily comprehensible in the early stages of her illness as an emotional reaction to her realization of them. It is not hard to understand how a girl of her age, appearance, and personality could become jealous of her sister's good health, seclusive and morose on account of her changed appearance, irritable in view of the pain which she suffered, and later depressed when she found that no one seemed capable of doing anything to alleviate her condition. As the illness progressed, however, other factors, as mentioned at the beginning of the paper, and more particularly the physiological abnormalities, came into greater prominence, and more and more clearly could be seen reflected in the psychological picture. Of these the most obvious were changes which occur frequently in hypertensive 
states, such as loss of the elasticity and adaptability of the personality in regard to all mental processes, the exaggeration of psychological features which were present before but either latent or less manifest, and depression with paranoid delusions and periods of confusion, panic, or excitement. This corresponds closely to the descriptions of psychoses in hypertensive states by such writers as Krapf (1937) and Meyer-Gross (1937). Other physiological disturbances must also exert an influence on the mental state, but in the present state of knowledge their effect cannot be assessed. In our case, no straightforward causal relationship between endocrine disorder and psychological symptomatology can be demonstrated. The features in evidence are the great emotional stress to which the patient was subjected, the change of personality, and the onset of a psychosis. The early symptoms are easily understood as a psychological response; the final symptoms are definitely of an organic kind, due to systemic disturbances which finally involved the brain and disturbed its function. It is, however, interesting to note that the psychological mechanisms, set going initially by psychological factors, were still at work in the final stages, colouring the contents of the mental state. This points to the importance of analysing in each case how far the symptoms can be explained on the psychological level, how far it is necessary to refer them to physical mechanisms. Of the latter the endocrine influence on mental function is one important aspect which needs much further investigation.

The endocrinologist, in his observations, has been concerned chiefly with the physical, organic consequences of endocrine upset, while the concomitant mental state, so necessary for further psychiatric understanding, is sometimes barely described, but the psychobiological approach is of practical importance and in the severer forms of humoral upset, such as Cushing's syndrome, its adoption would certainly lead to the eliciting of mental symptoms of greater or lesser degree and to increased knowledge of the factors responsible for the various syndromes.

We wish to thank Professor E. Mapother and Dr. Wooton for permission to use the clinical data ; Mr. Cairns for the operation notes; and Professor Golla and the staff of the Central Pathological Laboratory for some of the pathological investigations.

\section{Notes on Pathological Examination}

Summary of Macroscopic and Microscopic Findings.-Sero-fibrinous pleurisy (10 oz.) of left sac. Clear effusion (about 6 oz.) in right pleural sac. Fibrous adhesions uniting upper lobe of left lung to parietal pleura over fourth and fifth ribs. Chronic bronchiectasis and purulent broncho-pneumonic consolidation of lower lobe of left lung. Purulent broncho-pneumonia of upper lobe of right lung. Edema and congestion of remainder of lungs. Fatty degeneration of myocardium. Slight tortuosity of coronary arteries. Slight general atheroma. Back-pressure atrophy of centres of lobules and slight fatty degeneration of liver. Slight congestion of spleen. Mucous catarrh of stomach. Areas (up to $16 \mathrm{~cm}$. long) of discrete and confluent recent hæmorrhage and necrosis in mucosa of lower jejunum and upper ileum. Chronic Bright's disease (nephritis repens, type IV). Small ovaries containing microscopically a few corpora fibrosa and one Graafian follicle. Infantile uterus (cervix, $3.5 \mathrm{~cm}$. long ; body, $3.0 \mathrm{~cm}$. long). Uniform hyperplasia of cortex of supra- 
renal bodies. Normal colloidal thyroid gland. Four normal parathyroid glands. Cyst $(0.7 \mathrm{~cm}$. diam.) in head of otherwise normal pancreas. Glandular tissue in excess of adipose in thymus. Small pituitary gland in intact sella turcica showing perforation $(0.05 \mathrm{~cm}$. diam.) in its right lateral border corresponding to site of radon seed. No radon seeds found. Atrophy of right optic nerve, showing operative division immediately anterior to chiasma. Slight atrophy of frontoparietal convolutions. Healed fractures in costal ends of all right ribs and in lower left ribs. Osteoporosis (macroscopic) of ribs. Bodies of lumbar vertebræ easily cut with cartilage knife. No macroscopic evidence of osteoporosis in corticalis of femur. Petechiæ in skin of thorax. Faint diffuse brown pigmentation of skin of neck and less marked of face. Bruising of skin over anterior borders of both tibiæ. No striæ albicantes present. Excessive growth of hair in moustache and beard areas and over sides of cheeks. A greatly wasted woman with no local adiposity.

Pituitary gland, 0.4 gm. ; suprarenal bodies, 24.45 gm.; ovaries, 7.4 gm.; thyroid, $17.3 \mathrm{gm}$.; thymus, $11.05 \mathrm{gm}$.; parathyroid glands, $130 \mathrm{mg}$.; heart, 276 gm. ; kidneys, 248 gm. ; pancreas, $55 \cdot 5 \mathrm{gm}$.

The pituitary gland was divided horizontally and both halves were embedded together in paraffin. Serial sections stained with a modification of Mallory's acid-fuchsin anilin-blue method (Crooke and Russell (1935) ) show no adenoma. Around gaps where the radon seeds had been inserted in the anterior and right lateral borders of the anterior lobe there are broad zones where the cells have been completely destroyed, leaving an acellular collagenous stroma (Fig. 3). In the remainder of the anterior lobe there is a remarkable diminution in the number of basophil cells, most of which show the peculiar hyaline change described by Crooke (1935). The acidophil cells are abundant and their granules well preserved. The remainder of the gland appears normal. Sections in the coronal plane through the anterior part of the right and left hypothalamus show an area $(0.4 \times 0.3 \mathrm{~cm}$.) of complete necrosis in the optic chiasm, immediately to the right of the middle line. It extends ventrally to the pia and dorsally to the subependymal glia of the floor of the third ventricle. Surrounding this area is a fringe of compound granular corpuscles, and these are also sparsely scattered throughout the remainder of the chiasm. There is diffuse gliosis throughout the chiasm and many of the myelinated fibres have been destroyed except at the left border. The left supraoptic nucleus is well preserved, but many neurones have disappeared from the right.

\section{Comment}

This case is clearly an example of Cushing's syndrome. All the characteristic clinical features were present at some period of the illness, except cutaneous striæ, the absence of which suggests that the abdominal obesity was never great. Transient obesity was, however, noted in Case 1 of the report by Russell, Evans, and Crooke (1934), in which Cushing's syndrome was associated with a basophil adenoma of the pituitary in a male subject.

Pathological examination revealed no tumour in any of the endocrine glands. Several examples of the syndrome are, however, on record in which a similar negative result was obtained : Rutishauser (1933), Case 3 ; Kepler et al. (1934), Case 1; de Jongh (1935); Ulrich, quoted by Rasmussen (1936). Diffuse 
hypertrophy of the suprarenal cortex and atrophy of the sex organs were however, present, and have been recorded in similar cases. Further the hyaline change in the cytoplasm of the basophil cells in the pituitary gland was well marked, and this in our experience is so conspicuous and constant a finding in other examples of Cushing's syndrome that it may be regarded as a diagnostic feature. The great paucity of basophil cells in the pituitary is difficult to explain. Without confirmatory evidence it can hardly be attributed to a specific selective effect of the radon. On the other hand, it has been observed (Crooke, 1935) that the number of basophil cells in the acini of the anterior lobe varies greatly in different examples of the syndrome. In Case 2 of his series it was noted that the reduction in the number of basophil cells was so great that the sections were comparable in this respect to the pituitary gland in Addison's disease. It is likely, therefore, that the extreme reduction in the present case is independent of the insertion of radon seeds.

Dr. Russell was working on behalf of the Medical Research Council.

\section{REFERENCES}

Birnbaum, K. (1930). Handwörterbuch der medizinischen Psychologie. Leipzig. 1930. Crooke, A. C., and Russell, D. S. (1935). J. Path. Bact., 40, 255.

Crooke, A. C. (1935). Ibid., 41, 339.

Cushing, H. (1932). Johns Hopk. Hosp. Bull., 50, 137.

De Jongh, C. L. (1935). Ned. Tijdschr. Geneesk., 79, 1,805.

Hoskins, R. G. (1934). The Problem of Mental Disorder, p. 234. New York.

Kepler, E. J., et alia (1934). Proc. Staff. Meet. Mayo Cl., 9, 169.

Kraepelin, E., and Lange, I. Psychiatrie. Leipzig, 1927.

Krapf, E. (1937). J. ment. Sc., 83, 346, 534.

Lescher, F. G., and Robb Smith, A. H. T. (1935). Quart. J. Med., 4, 23.

Mayer-Gross, W. (1937). J. ment. Sc., 83, 346, 551 .

Pardee, I. (1937). Bull. neurol. Inst. N.Y., 6, 183.

Rasmussen, A. T. (1936). Endocrinology, 20, 673.

Russell, D. S., Evans, H., and Crooke, A. C. (1934). Lancet, 2, 240.

Rutishauser, E. (1933). Dtsch. Arch. klin. Med., 175, 640. 


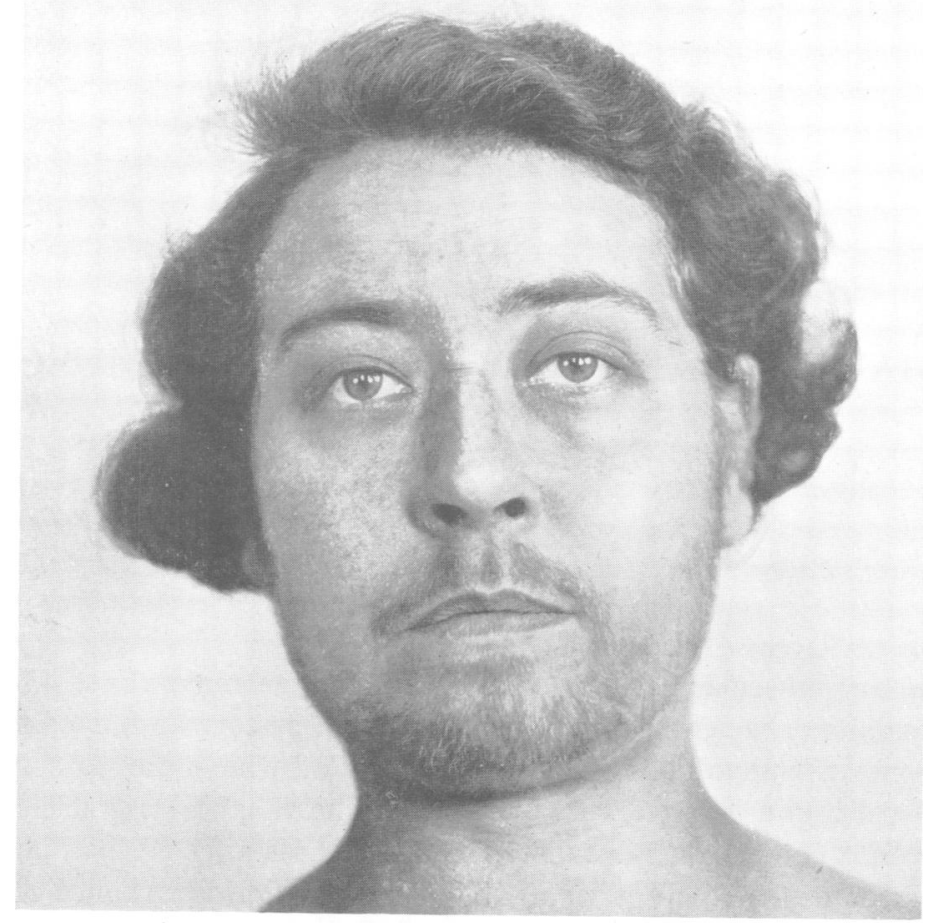

Fig. 2.-Patient after onset of Cushing's Syndrome.

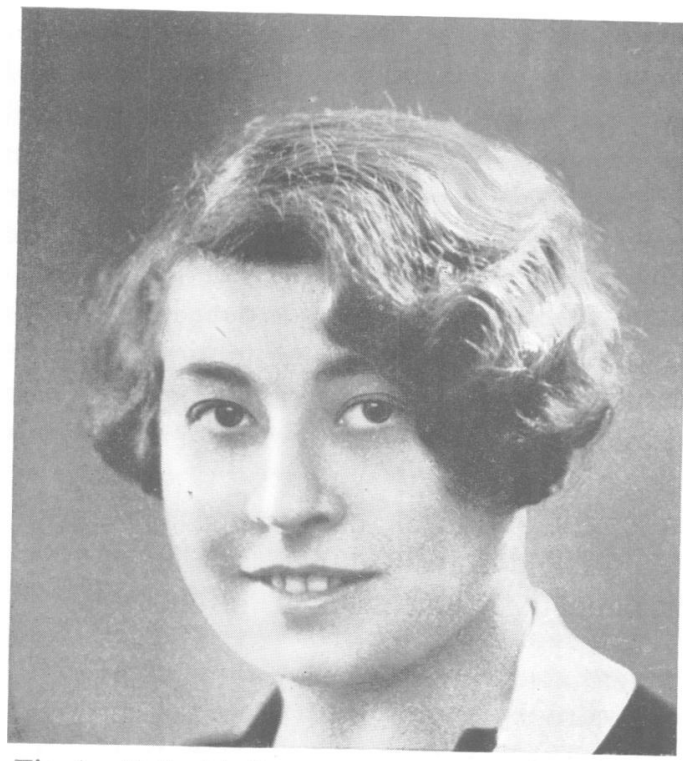

Fig. 1.-Patient before onset of Cushing's Syndrome.

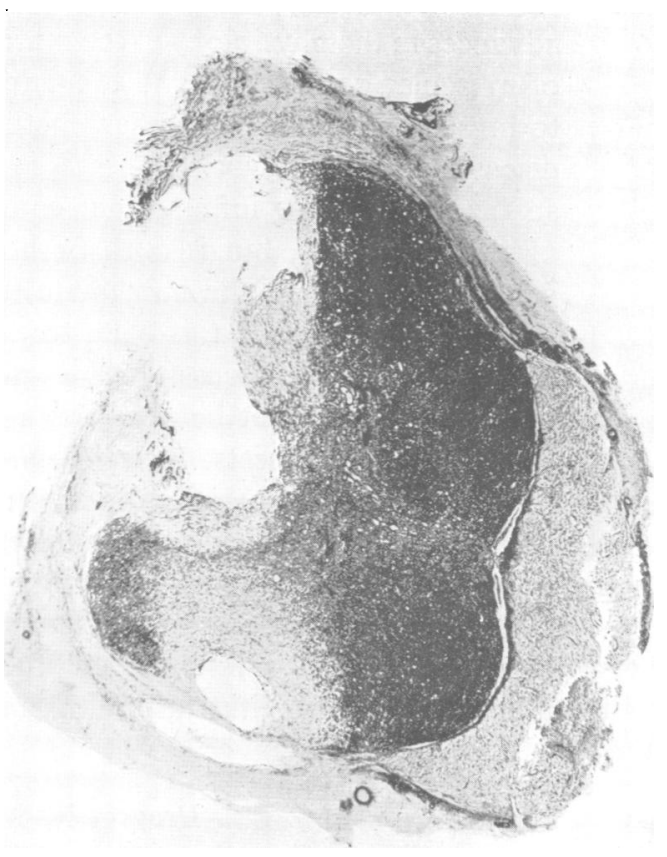

Fig. 3.- Horizontal section of pituitary gland showing areas of destruction by radon seeds. Mallory's acidfuchsin anilin-blue ; $\times 6 \cdot 5$. 